OPEN ACCESS

Citation: Timir Baran Jha, Biplab Kumar Bhowmick, Partha Roy (2021)Analysis of CMA-DAPI bands and preparation of fluorescent karyotypes in thirty Indian cultivars of Lens culinaris. Caryologia 74(2): 65-77. doi: 10.36253/caryologia-919

Received: April 24, 2020

Accepted: May 19, 2021

Published: October 08, 2021

Copyright: $@ 2021$ TimirBaran Jha, Biplab Kumar Bhowmick, Partha Roy. This is an open access, peer-reviewed article published by Firenze University Press (http://www.fupress.com/caryologia) and distributed under the terms of the Creative Commons Attribution License, which permits unrestricted use, distribution, and reproduction in any medium, provided the original author and source are credited.

Data Availability Statement: All relevant data are within the paper and its Supporting Information files.

Competing Interests: The Author(s) declare(s) no conflict of interest.

ORCID

TBJ: 0000-0003-0900-8167

BKB: 0000-0001-6029-1098

\section{Analysis of CMA-DAPI bands and preparation of fluorescent karyotypes in thirty Indian cultivars of Lens culinaris}

\author{
Timir Baran Jha ${ }^{1, *}$, Biplab Kumar Bhowmick ${ }^{2}$, Partha Roy ${ }^{1}$ \\ ${ }^{1}$ Department of Botany, Maulana Azad College, Kolkata -700013, West Bengal, India \\ ${ }^{2}$ Department of Botany, Scottish Church College, 1\&3, Urquhart Square, Kolkata- \\ 700006, West Bengal, India \\ *Corresponding Author. E-mail: tbjha2000@yahoo.co.in
}

\begin{abstract}
India holds a significant rank in production and consumption of the age old protein rich crop Lentil with only one cultivated species and a large number of phenotypically similar cultivars. The need for a reliable and cost effective method of genetic characterization to unravel differences within the Lentil cultivars was felt. The present paper adopted EMA based chromosome preparation followed by staining with two contrasting fluorochrome dyes CMA and DAPI that bind directly to GC and AT rich heterochromatic segments on chromosomes. Analysis of fluorochrome banding pattern furnished a comparative account of genetic diversity within the cultivars that could not be achieved by traditional karyotyping. The marker pair of nucleolar chromosomes ( $4^{\text {th }}$ and $3^{\text {rd }}$, majorly) occupied a pivotal position to intensify differences between cultivars in terms of banding patterns around secondary constrictions, suggestive of yet unknown variation in heterochromatin composition. Our study has strengthened genetic background and relationships of Lentil cultivars. We observed certain types of unusual fluorochrome bands that put forward the exclusivity of Indian germplasm and have questioned the mainstream heterochromatin elements of plant chromosomes captured by CMA-DAPI stains. The comprehensive fluorescent karyotypes of $30 \mathrm{~L}$. culinaris Medik. cultivars prepared for the first time, serve as an archetype for the benefit of future breeding programmes.
\end{abstract}

Keywords: lentils, CMA-DAPI, chromosomal bands, fluorescent karyotype, heterochromatin.

\section{INTRODUCTION}

Lentil is one of the richest protein containing domesticated ancient crop with only one globally cultivated species Lens culinaris Medik. India is the second highest producer and biggest consumer of Lentils. The genus belongs to the largest subfamily (Papilionoideae) of Fabaceae (Azani et al. 2017), along with many economically important genera producing pulses and beans. Being the single cultivated species, large number of cultivars is in cultivation in our country. The characterization of Indian germplasm is 
needed to sustain conservation and programmable utilization of resources. Chromosomal characterization is a cost effective method to provide foundational information on the genome and genetic conservation for any future breeding program of particular crop plants. Cytogenetic studies of Indian Lentils through conventional method failed to provide uniformity on chromosome morphometric parameters (Bhattacharjee 1953; Sharma and Mukhopadhyay 1963; Sinha and Acharia 1972; Naithani and Sarbhoy 1973; Lavania and Lavania 1983; Nandanwar and Narkhede 1991). On the other hand, we have published detailed karyotype analysis of more than thirty L. culinaris cultivars obtained from the Indian Institute of Pulses (Jha et al. 2015, 2017; Jha and Halder 2016) through EMA based Giemsa staining method. Our results were found to have near similarities with the results obtained by Ladizinsky (1979). However, Lens chromosomes $(2 \mathrm{n}=14)$ are nearly similar in morphology. Considering the status of research, we question i) is there any karyotype variability across cultivars beyond chromosome number, morphology and ploidy? ii) is it possible to find visible chromosomal landmarks in accordance with the germplasm diversity? and iii) whether we can step forward towards molecular kayotype database for Indian Lentils. As EMA based chromosome analysis (Fukui 1996) is the basis of molecular cytogenetics, we decided to carry forward our work with two contrasting fluorescent stains DAPI and CMA on the same cultivars. Having affinity towards specific base pairs of DNA, these fluorescent dyes reliably identify heterochromatin rich sectors on chromosomes, differentiate morphologically alike chromosomes and improve karyotype characterization (Schweizer 1976; Guerra et al. 2000; Yamamoto 2012; Weiss-Schneeweiss and Schneeweiss 2013). So, our objective is to address chromosomal behavior after application of base specific fluorochromes and compile cultivar specific fluorescent banding profiles. The present paper considers a fluorescent karyotype dataset of 30 Indian L. culinaris cultivars for the first time, as an important kit for Lentil breeders and genome researchers.

\section{MATERIALS AND METHODS}

\section{Chromosome preparation and fluorochrome staining}

The fluorescent karyotype analysis was carried out on 30 cultivars of Lens culinaris presented in Table 1. Except for two (Barasat, Micro type and Barasat, Macro type, Table 1), all the cultivars of Lentil were obtained from the Indian Institute of Pulse Research (IIPR), Kanpur. Germination of seeds and chromosome pro- cessing through enzymatic maceration and air drying (EMA) was carried out as per our earlier protocol (Jha and Yamamoto 2012; Jha et al. 2015, 2017, 2020). For fluorescent staining with DAPI and CMA, we followed our protocol (Jha 2019) with required modifications. For DAPI staining, slides were kept for $30 \mathrm{~min}$ in McIlvaine buffer, stained with $0.1 \mu \mathrm{g} \mathrm{ml}^{-1}$ solution of DAPI for $10 \mathrm{~min}$, counterstained with $0.25 \mathrm{mg} / \mathrm{ml}$ of Actinomycin $\mathrm{D}$ (AMD) for $15 \mathrm{~min}$ and then mounted in nonfluorescent glycerol and observed under Carl Zeiss Axio Lab A1 fluorescence microscope using Carl Zeiss DAPI filter cassette. Chromosome images were captured with CCD camera attached with microscope. The slides were destained and air-dried. The same slides were placed in McIlvaine buffer for $30 \mathrm{~min}$ followed by incubation in McIlvaine buffer with $5 \mathrm{mM} \mathrm{MgCl}_{2}$ for 10 mins and then stained with $0.1 \mathrm{mg} \mathrm{ml}^{-1} \mathrm{CMA}$ solution for 45-50 mins. The slides were again washed in McIlvaine buffer with $5 \mathrm{mM} \mathrm{MgCl}{ }_{2}$ and finally mounted with non-fluorescent glycerol and kept for maturation at $4^{\circ} \mathrm{C}$ for $48-72 \mathrm{hrs}$. CMA stained slides were observed under the abovementioned fluorescence microscope fitted with Carl Zeiss FITC filter cassette, images captured with attached CCD camera and signals were analyzed using the software Prog Res 2.3.3.

\section{Statistical analysis of karyotype relations}

Karyotype relations among the cultivars was evaluated with the help of cluster analysis for data matrix normalization by unweighted pair group method with arithmetic averages (UPGMA) based on Euclidean distance using Info Stat 2017d (free version). Here, only the fluorochrome banding pattern of the cultivars viz. types and numbers of CMA and DAPI bands were utilized to draw the phenogram.

\section{RESULTS}

Fluorochrome banding pattern in cultivars of L. culinaris Medik.

Somatic chromosome analysis of the 30 Lentil cultivars based on fluorescence banding patterns has provided an interesting catalogue of chromosome diversity. The chromosomes took up DAPI stain within 10 minutes of incubation while the incubation time for CMA staining was about 45-50mins. The same CMA and DAPI staining protocol was followed for all the 30 cultivars of $L$. culinaris. Interestingly, we have obtained different types of DAPI and CMA banding patterns within the studied 

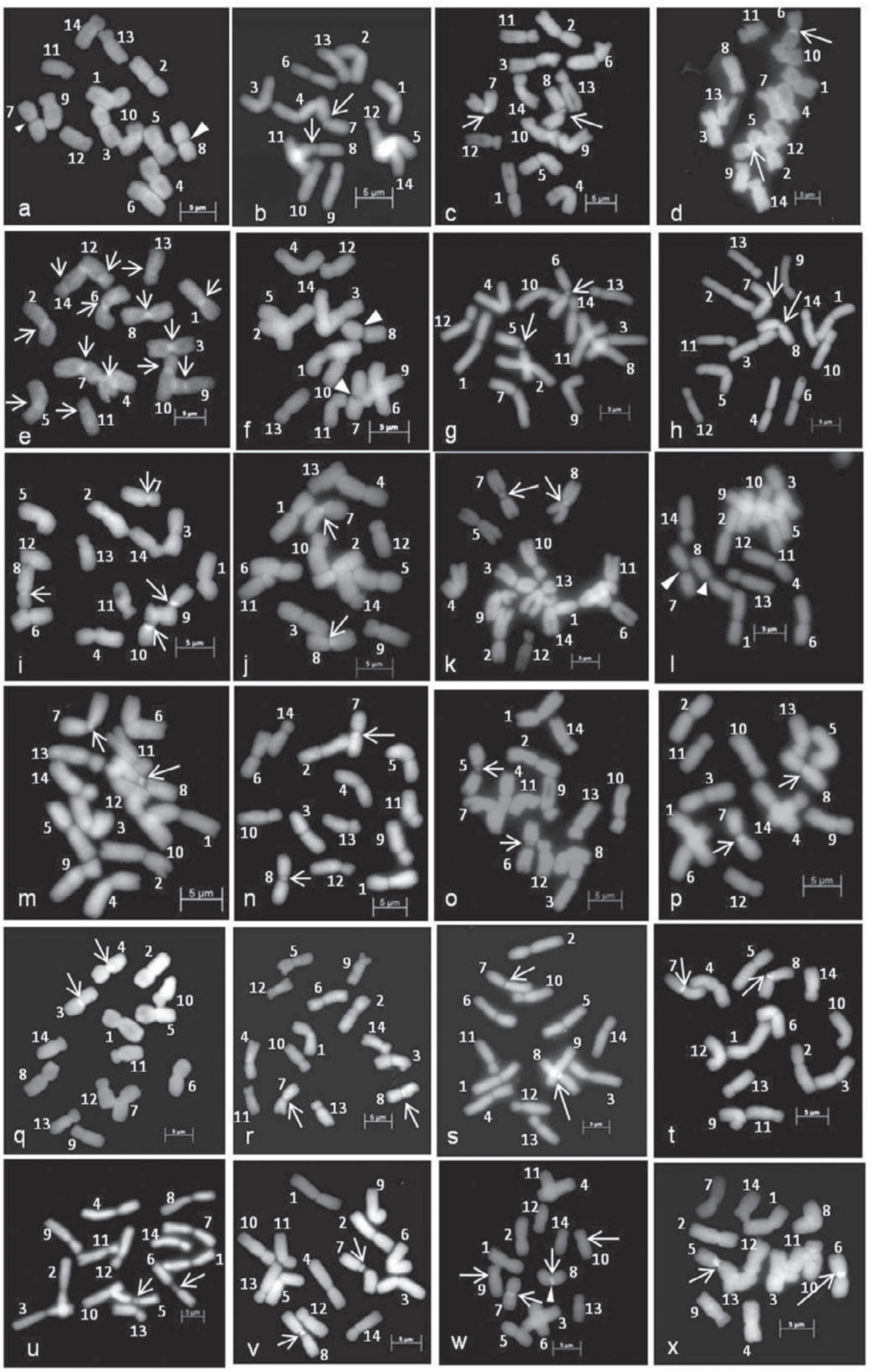

Figure 1. Somatic metaphase chromosomes of Lens culinaris cultivars stained with CMA: (a) DPL-15, (b) DPL-62, (c) IPL-81, (d) IPL-406, (e) IPL-316, (f) JL-1, (g) HUL-57, (h) KLS-210, (i) EC-70394, (j) EC-70403, (k) EC-70404, (l) EC-78452, (m) EC-78455, (n) EC-78461, (o) EC-78475, (p) EC-78498, (q) EC-78542-A, (r) EC-223188, (s) EC - 255491, (t) EC-267526, (u) EC-267569-A, (v) EC-267590, (w) EC-267877, (x) Barasat Micro type. White arrows indicate CMA ${ }^{+}$bands and arrowheads indicate CMA ${ }^{0}$ bands. Bars $5 \mu \mathrm{m}$. 
cultivars. At least 10 plates stained with DAPI and CMA for each cultivar was considered for analysis of banding types. Secondary constriction marked the nucleolar organizing region (NOR) of most of the cultivars, showing $\mathrm{CMA}^{+}$bands with different intensities while some NORs remained neutral and termed $\mathrm{CMA}^{0}$ as per Barros e Silva and Guerra (2010). DAPI staining in most of the cultivars resulted a clear gap (DAPI-) corresponding to $\mathrm{CMA}^{+}$band. However, few exceptional cultivars yielded $\mathrm{DAPI}^{+}$band in the NOR regions. Based on the CMA and DAPI fluorescent banding, we have categorized following types of somatic chromosomes. The chromosomes with $\mathrm{CMA}^{+} / \mathrm{DAPI}^{-}$band in the nucleolar region is termed type 'A'. Type ' $\mathrm{B}$ ' has $\mathrm{CMA}^{+} / \mathrm{DAPI}^{-}$nucleolar constriction followed by a $\mathrm{DAPI}^{+} / \mathrm{CMA}^{0}$ band below centromere. The ' $\mathrm{C}$ ' type nucleolar chromosome has a distinct $\mathrm{CMA}^{+} / \mathrm{DAPI}^{+}$secondary constriction. The fourth type ' $\mathrm{D}$ ' has neutral CMA band in secondary constriction. Chromosomes with centromeric $\mathrm{CMA}^{+} / \mathrm{DAPI}^{0}$ bands are termed type ' $\mathrm{E}$ ' while those with centromeric $\mathrm{DAPI}^{+} / \mathrm{CMA}^{0}$ bands are termed type 'F'. Type 'G' chromosome contains intercalary $\mathrm{DAPI}^{+} / \mathrm{CMA}^{0}$ band. The chromosomes having no detectable bands were termed as type ' $H$ '. Distribution of different types of fluorochrome bands among the cultivars is summarized in Table 1. A detailed analysis of the fluorochrome stained metaphase plates (Figures 1-3) was carried out to formulate the diagrammatic fluorescent karyotypes of the 30 cultivars under study (Figures 4 and 5).

CMA-DAPI banding patterns have revealed that in majority of L. culinaris cultivars (Table 1), the marker secondary constrictions with $\mathrm{CMA}^{+}$signals are present in the $4^{\text {th }}$ pair of chromosomes. However, the same in some cultivars are present in the $3^{\text {rd }}$ and exceptionally in the $5^{\text {th }}$ and $2^{\text {nd }}$ pairs, as in two cultivars (EC-70394, EC-78542-A). The most abundant $\mathrm{CMA}^{+}$satellites (type A chromosomes) are found among $50 \%$ of the presently studied cultivars. In addition to $\mathrm{CMA}^{+}$satellites, existence of type $\mathrm{B}$ chromosomes is found in 8 different cultivars (HUL-57, EC-70403, EC-78542-A, EC-267526, EC 267877, Barasat Micro type, PL -1406, EC -78410, Table 1) and type D chromosomes in 5 different cultivars (DPL15, JL-1, EC-78452, EC - 70306, EC - 78473, Table 1). Of special mention, are the two cultivars (EC-70404, EC267569-A, Table 1) with $\mathrm{CMA}^{+} / \mathrm{DAPI}^{+}$satellite (type C chromosome). Three cultivars (IPL -316, EC-70394, EC 267877) had centromeric $\mathrm{CMA}^{+}$bands (type E) (Table 1). One of them (IPL -316) shows centromeric $\mathrm{CMA}^{+}$bands (type E) in every chromosome except the nucleolar pair (Table 1). On the other hand, EC -78410 shows intense centromeric $\mathrm{DAPI}^{+}$bands (type F) in all non-nucleolar chromosome pairs (Table 1). Centromeric $\mathrm{DAPI}^{+}$bands are consistently found in the $2^{\text {nd }}$ or the $3^{\text {rd }}$ pair of chromosomes in 5 cultivars (HUL-57, EC-70403, EC-267526, Barasat, Macro type, PL -1406, Table 1). Intercalary $\mathrm{DAPI}^{+}$band (type G) is seen only in IPL-406 (Table 1).

\section{Comparative statistical assessment of fluorochrome banding pattern}

Statistical evaluation of karyotype relations among the 30 Lentil cultivars was carried out using Euclidean distance matrix on the basis of CMA and DAPI bands. The UPGMA phenogram presented relative karyotype affinities and distances with a cophenetic correlation of 0.986 as a good fit between the cophenetic value matrix and the average Euclidean distance matrix (Figure 6). There are three separate groups in the UPGMA phenogram of which Group I consisted of cultivars that do not have close affinity with each other (Figure 6). Within this group, EC -78410 and IPL -316 have fluorescent banding pattern that are in contrast to each other. Also, existence of intercalary $\mathrm{DAPI}^{+}$band makes IPL-406 distinct, placed at the extreme end of the phenogram. The next noticeable cultivars are EC-70404 and EC-267569-A with $\mathrm{CMA}^{+} / \mathrm{DAPI}^{+}$secondary constriction (Table 1) (Figure 6). The Group II is large, composed of three subgroups mainly differentiated by nucleolar banding pattern in their marker chromosomes. The first subgroup comprised of 5 cultivars with neutral CMADAPI bands in their satellites (type D) (Table 1, Figure 6). The second subgroup is largest, comprising of 13 cultivars with $\mathrm{CMA}^{+} / \mathrm{DAPI}^{-}$satellite (type A). Here, two cultivars (EC-70394 and Barasat, Macro type) show little distance from rest of the cultivars, because of different types of centromeric bands (Table 1, Figure 6). The third subgroup comprises of 7 cultivars with 'B' type nucleolar chromosomes. This subgroup shows heterogeneity because of variations in centromeric bands (Table 1, Figure 6).

\section{DISCUSSION}

Cytogenetics of L.culinaris is traditionally acknowledged for species delimitations, crossing behavior, conservation and utilization of plant genetic resources (Ladizinsky 1979; Tadmor et al. 1987; Ladizinsky et al. 1990; Ladizinsky 1999; Mishra et al. 2007). With the present approach, we have entered the modern karyotyping system to study chromosomal specialization in Indian Lentils. The diversity of fluorescent karyotypes can be indisputably attributed to the differences in underlying chromosomal heterochromatin of the samples since i) 

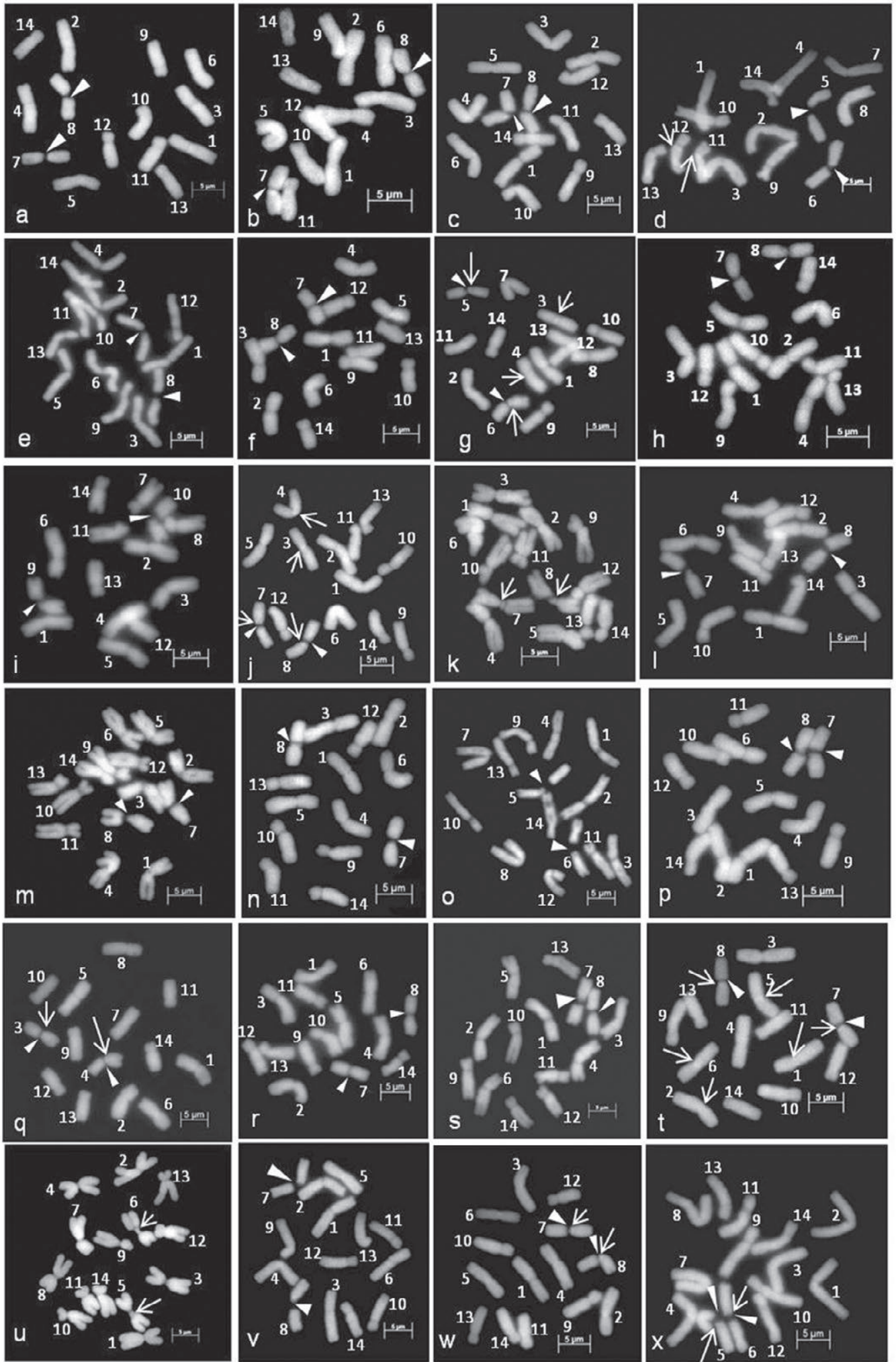

Figure 2. Somatic metaphase chromosomes of Lens culinaris cultivars stained with DAPI: (a) DPL-15, (b) DPL-62, (c) IPL-81, (d) IPL-406, (e) IPL-316, (f) JL-1, (g) HUL-57, (h) KLS-210, (i) EC-70394, (j) EC-70403, (k) EC-70404, (l) EC-78452, (m) EC-78455, (n) EC-78461, (o) EC-78475, (p) EC-78498, (q) EC-78542-A, (r) EC-223188, (s) EC - 255491, (t) EC-267526, (u) EC-267569-A, (v) EC-267590, (w) EC-267877, (x) Barasat Micro type. White arrows indicate DAPI ${ }^{+}$bands and arrowheads indicate DAPI and DAPI ${ }^{\circ}$ bands. Bars $5 \mu \mathrm{m}$. 

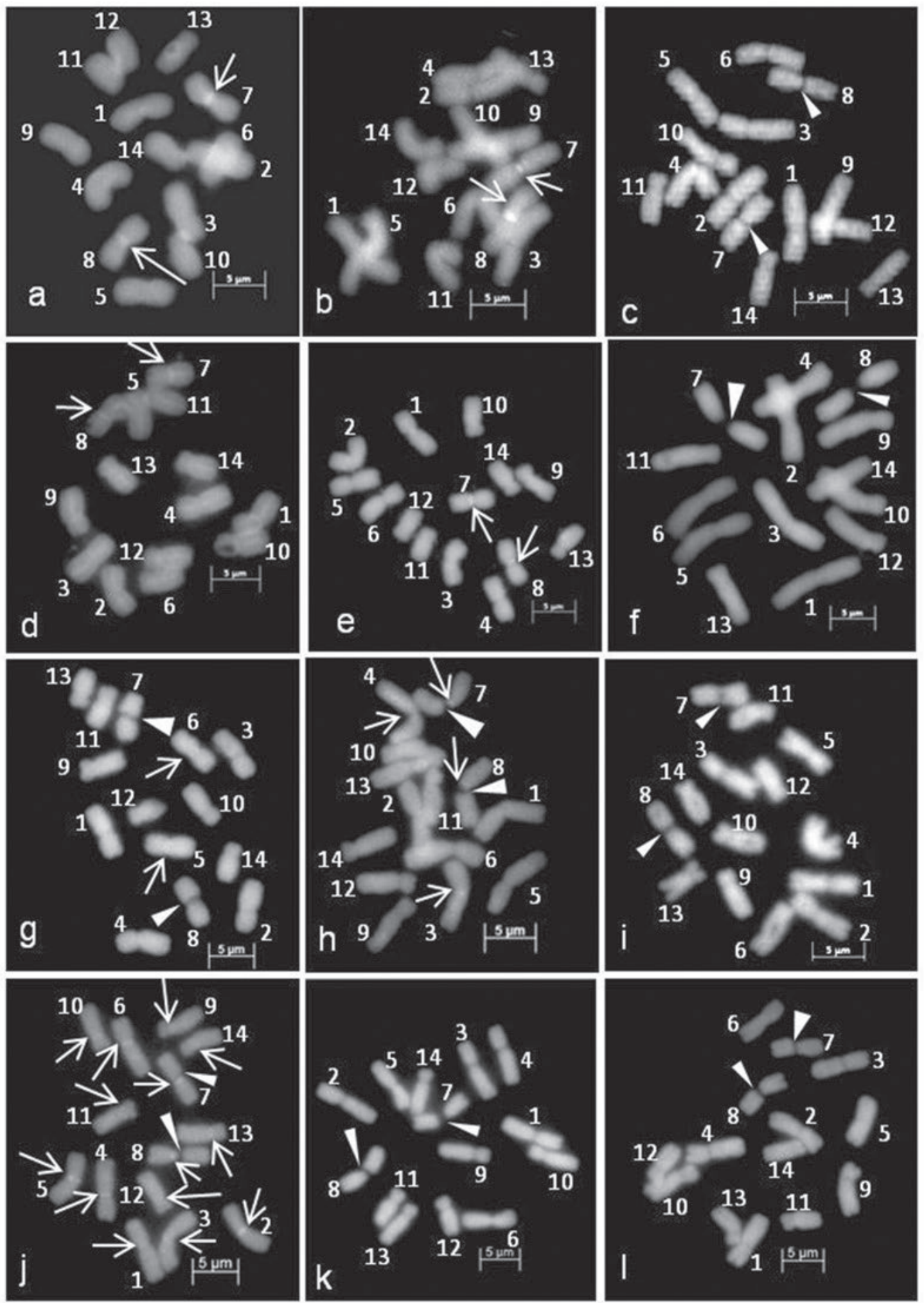

Figure 3. Somatic metaphase chromosomes of Lens culinaris cultivars. CMA stained plates: (a) Barasat Macro type, (b) PL-1406, (c) EC-70306, (d) EC -78410, (e) EC-78451-A, (f) EC-78473. DAPI stained plates: (g) Barasat Macro, (h) PL-1406, (i) EC-70306, (j) EC -78410, (k) EC-78451-A, (l) EC-78473. White arrows indicate CMA ${ }^{+}$or DAPI ${ }^{+}$bands and arrowheads indicate CMA ${ }^{0}$ or DAPI $^{0}$ and DAPI bands. Bars $5 \mu \mathrm{m}$. 


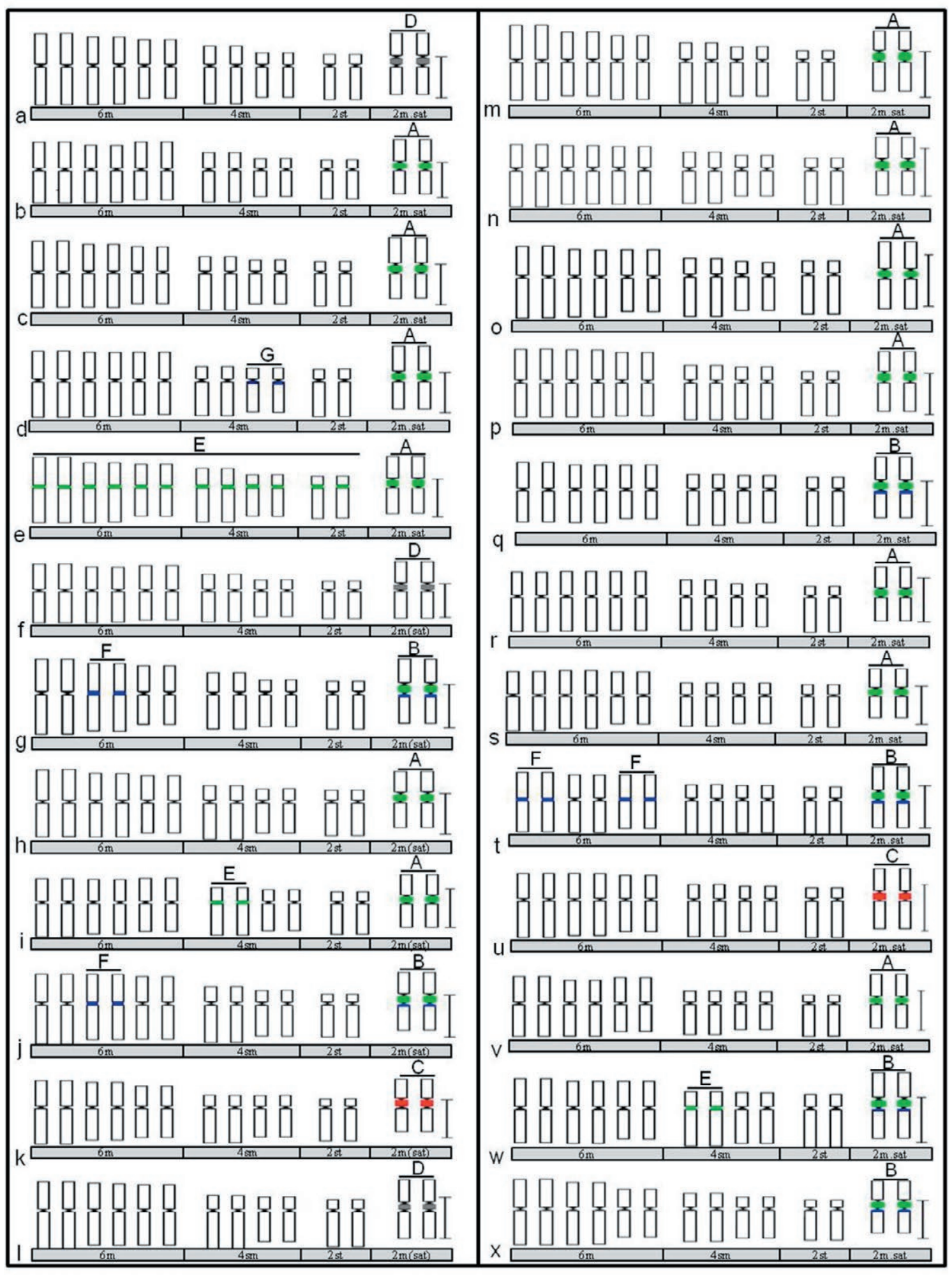

Figure 4. Fluorescent ideograms of Lens culinaris cultivars based on CMA/DAPI banding pattern: (a) DPL-15, (b) DPL-62, (c) IPL-81, (d) IPL-406, (e) IPL-316, (f) JL-1, (g) HUL-57, (h) KLS-210, (i) EC-70394, (j) EC-70403, (k) EC-70404, (l) EC-78452, (m) EC-78455, (n) EC-78461, (o) EC-78475, (p) EC-78498, (q) EC-78542-A, (r) EC-223188, (s) EC - 255491, (t) EC-267526, (u) EC-267569-A, (v) EC-267590, (w) EC-267877, (x) Barasat Micro type. CMA ${ }^{+}$, DAPI ${ }^{+}, \mathrm{CMA}^{+} / \mathrm{DAPI}^{+}$and $\mathrm{CMA}^{0}$ bands are highlighted with green, blue, red and grey colors on the chromosomes, respectively and the types are indicated above the chromosome diagrams. Bars $5 \mu \mathrm{m}$ 


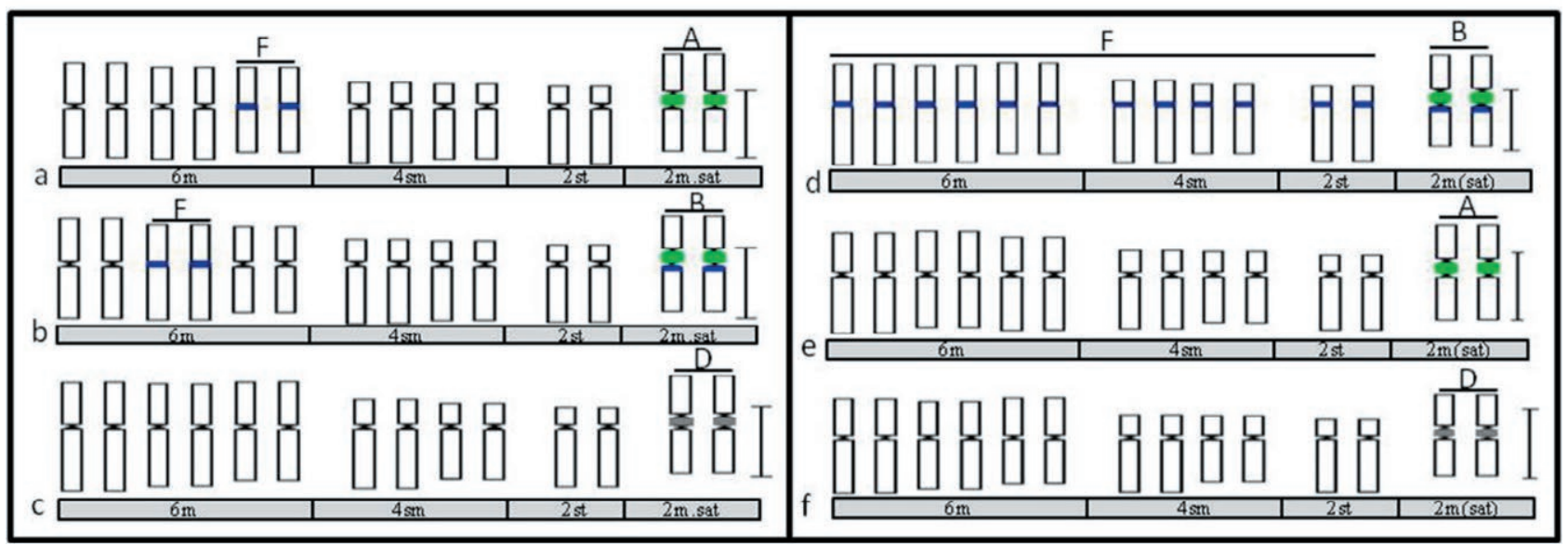

Figure 5. Fluorescent ideograms of Lens culinaris cultivars based on CMA/DAPI banding pattern: (a) Barasat Macro type, (b) PL-1406, (c) EC-70306, (d) EC -78410, (e) EC-78451-A, (f) EC-78473. CMA ${ }^{+}, \mathrm{DAPI}^{+}, \mathrm{CMA}^{+} / \mathrm{DAPI}^{+}$and $\mathrm{CMA}^{0}$ bands are highlighted with green, blue, red and grey colors on the chromosomes, respectively and the types are indicated above the chromosome diagrams. Bars $5 \mu \mathrm{m}$.

we have applied the same fluorochrome staining protocol for every cultivar, ii) the method is repeated a number of times before ascertaining banding pattern in a cultivar and iii) at least 5 best metaphase plates of each cultivar with scorable signals were considered for establishing the fluorescent karyotype.

Considering the nature of nucleolar chromosomes, molecular banding technique has shed light on chromosomal landmarks and possible differences in NORs that were previously found to be similar in Lens (Mehra et al. 1986; Jha et al. 2015, 2017; Jha and Halder 2016). The marker nucleolar chromosomes $\left(4^{\text {th }}\right.$, along with the $3^{\text {rd }}, 2^{\text {nd }}$ and $5^{\text {th }}$ in few cases) have been confirmed with characteristic CMA-DAPI signals, corroborating to our previous report (Jha et al. 2017). The $\mathrm{CMA}^{+}$signals are generally accepted as the GC heterochromatic elements of the NORs in plant groups (Guerra et al. 2000; Barros e Silva and Guerra 2010; Yamamoto 2012; Olanj et al. 2015) and so in Papilionoids such as Vicia (Fuchs et al. 1998), Cicer (Galasso et al. 1996) and Crotalaria (Mondin and Aguiar-Perecin 2011). Previously, 18S-5.8S-25S rDNA probes had been localised in a single pair of $L$. culinaris, near the centromere (Balyan et al. 2002), corroborating to the observation of $\mathrm{CMA}^{+}$signals in our present study. However, we found that the intensity of the nucleolar CMA signals (type A) varies in certain cultivars, suggesting differences in NORs that influence affinity towards the stain. Intraspecific rDNA variation has been thoroughly worked out in Phaseolus (Moscone et al. 1999; Pedrosa-Harand et al. 2006) and Vigna (Bortoleti et al. 2012; She et al. 2015, 2020) of Papilionoideae. A number of factors such as transposition, unequal crossing over, inversion or locus duplication, had been suggested to drive NOR variation in plant groups, including Papilionoideae (Moscone et al. 1999; Chung et al. 2008; Raskina et al. 2008). We consider similar possibilities in the Indian Lentils, subject to future confirmation by AgNOR staining or rDNA FISH.

The type $\mathrm{D}$ chromosomes have satellites that respond indifferently to the CMA stain. The $\mathrm{CMA}^{0}$ satellites indicate GC neutral nature of heterochromatin (Barros e Silva and Guerra 2010). The type D satellites are in sharp contrast to type A bands, marking cultivar distinction. The other unusual type was the $\mathrm{CMA}^{+} /$ $\mathrm{DAPI}^{+}$satellites (type C). Previously, the $\mathrm{CMA}^{+} / \mathrm{DAPI}^{+}$ satellites were suggested to be a 'less common' or 'rare' type of heterochromatin (Barros e Silva and Guerra 2010), breaking the generality of GC rich composition of plant NORs (Schweizer 1976; Guerra et al. 2000). We document the occurrence of $\mathrm{CMA}^{+} / \mathrm{DAPI}^{+}$satellites for the first time in Lens of Papilionoideae. Co-localized $\mathrm{CMA}^{+} / \mathrm{DAPI}^{+}$satellites are so far reported in Allium nigrum (Maragheh et al. 2019) and Cestrum (Fernandes et al. 2009). It is difficult to ascertain the heterochromatin composition of this type. There is a possibility of having AT and GC rich segments to be placed so close that the different chromatin bands cannot be distinguished in condensed mitotic chromosomes (Maragheh et al. 2019). However, nucleolar heterochromatin composition of Indian Lens culinaris displays considerable variation, perhaps due to enormous cultivation practice and artificial hybridization, which is a yet unaddressed field of study.

Cultivar specific differences were also accentuated by the non-nucleolar $\mathrm{DAPI}^{+}$and $\mathrm{CMA}^{+}$bands. The type E centromeric CMA bands are unique type 


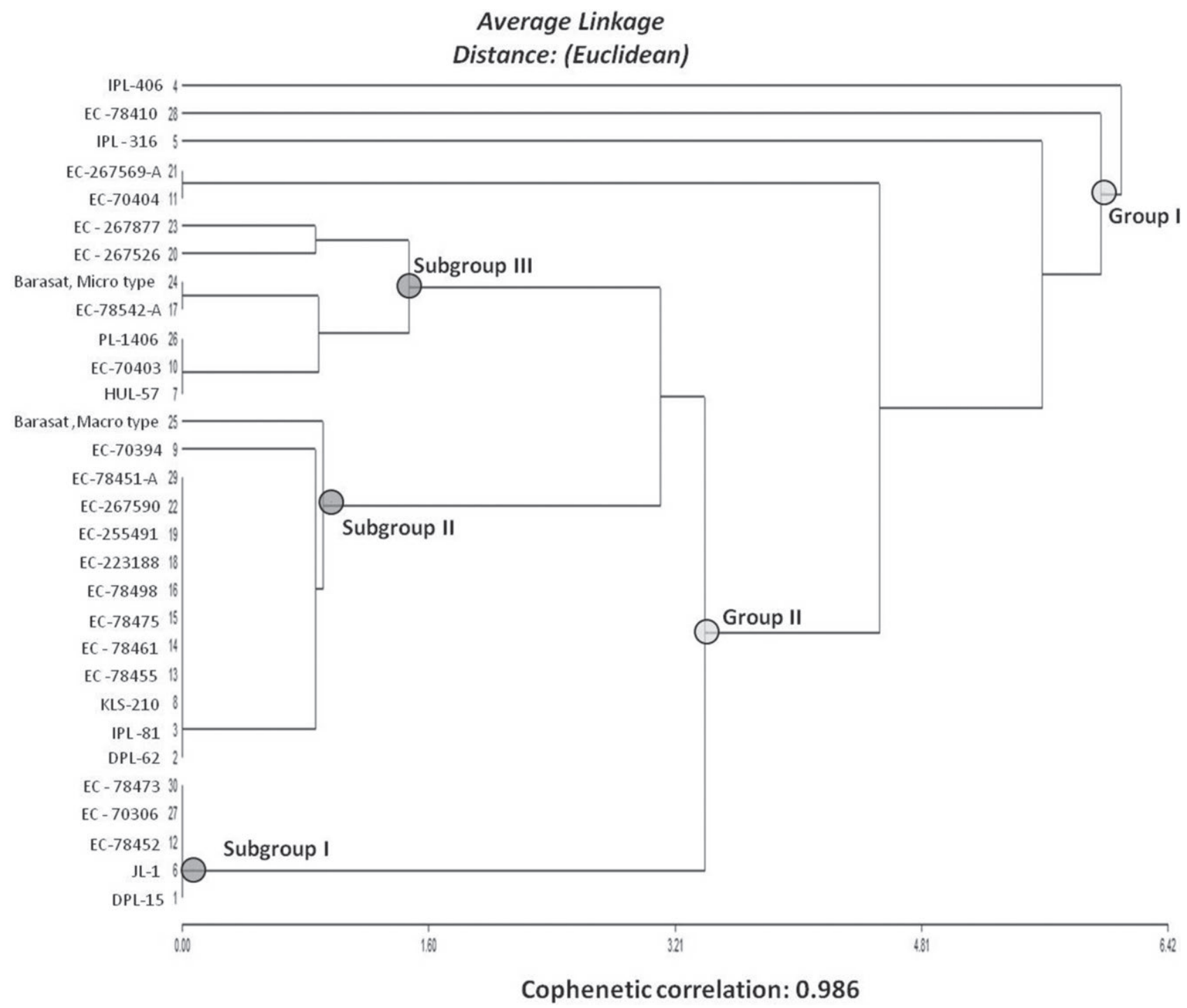

Figure 6. UPGMA dendrogram derived from average Euclidean distance based on fluorochrome banding pattern of 30 Indian Lentil cultivars, cultivar names in the left of their serial numbers.

of heterochromatin rarely reported in plants. However, non-nucleolar GC-rich heterochromatin was previously characterized in centromeric as well as pericentromeric regions of Papilionoid species belonging to Dioclea (Souza and Benko-Iseppon 2004), Psophocarpus (Chaowen et al. 2004), Crotalaria (Mondin and Aguiar-Perecin 2011), Vigna (Bortoleti et al. 2012; She et al. 2015, 2020), Phaseolus (Bonifácio et al. 2012), Lablab (She and Jiang 2015), and Canavalia (She et al. 2017). She et al. (2020) suggested the centromeric or pericentromeric GC- heterochromatin to be a relic of genomic evolution in the subfamily Papilionoideae. Other even rare heterochromatin blocks were the centromeric (type F), pericentromeric (type B) and intercalary (type G) DAPI bands, constituting landmarks to differentiate karyotypes of certain Lentil cultivars. Terminal or intercalary DAPI ${ }^{+}$ bands were documented in few plants (Vanzela and Guerra 2000; Divashuk et al. 2014), including few species of Cucurbitaceae (Bhowmick and Jha 2015, 2019). Terminal DAPI bands are found in Crotalaria (Mondin and Aguiar-Perecin 2011) of Papilionoideae. Centromeric DAPI bands are yet rare to encounter. However, in case of $L$. culinaris and related species, AT heterochromatic regions were mapped by repetitive sequence probe FISH (Galasso et al. 2001; Galasso 2003). Also in Papilionoideae, AT rich heterochromatin at centromere and pericentromeric regions are reported in Vigna (Bortoleti et al. 2012; She et al. 2020), Lablab (She and Jiang 2015) and 


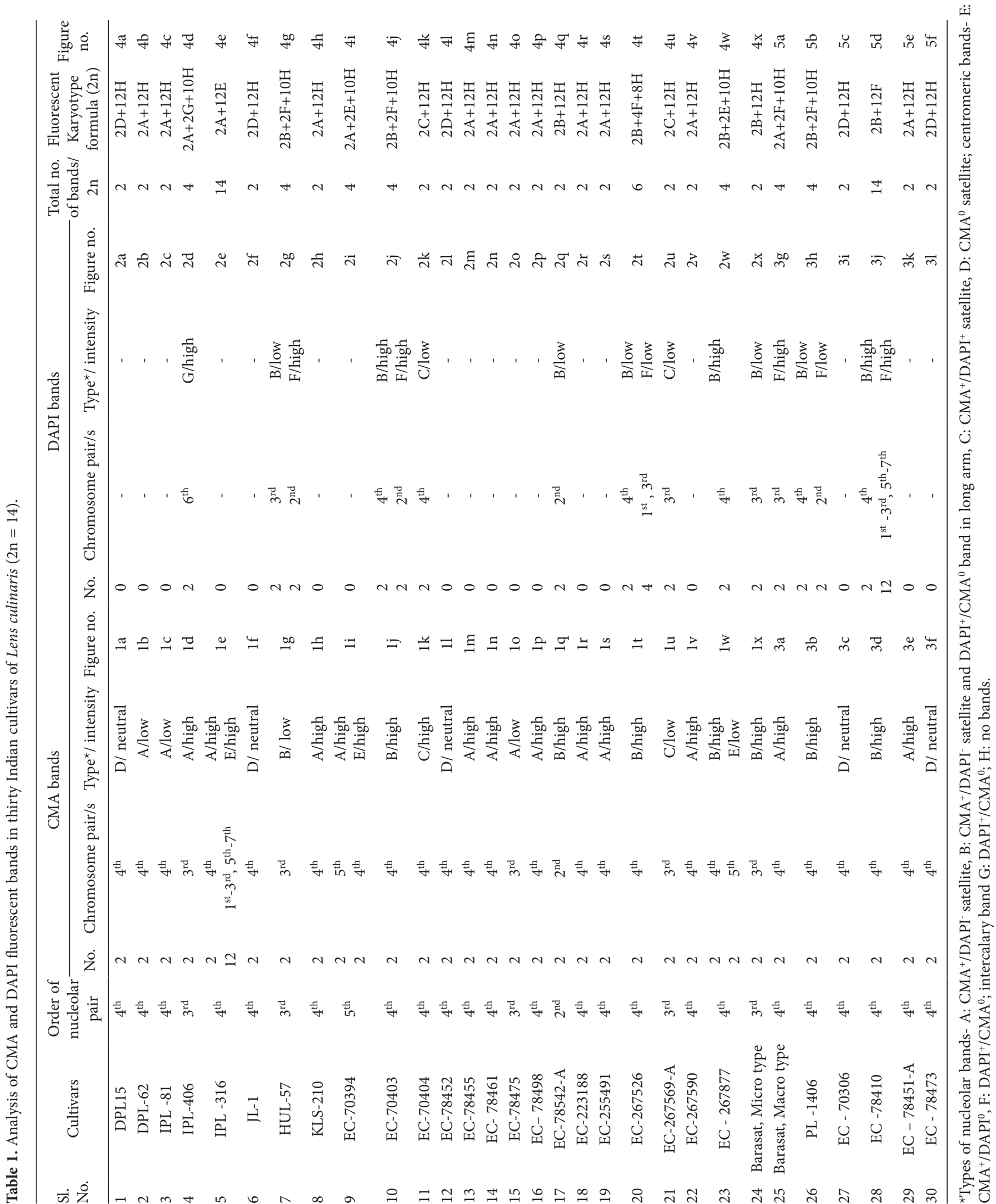


Arachis (Silvestri et al. 2020). Nonetheless, occurrence of centromeric $\mathrm{CMA}^{+}$or $\mathrm{DAPI}^{+}$bands along with nucleolar $\mathrm{CMA}^{+} / \mathrm{DAPI}^{+}$or $\mathrm{CMA}^{0}$ bands certainly advocate atypical heterochromatin composition in Lens. The non-uniform composition and rearrangements of heterochromatin had been observed repeatedly in Papilionoideae species (Moscone et al. 1999; Souza and Benko-Iseppon 2004; Pedrosa-Harand et al. 2006; Mondin and AguiarPerecin 2011; She et al. 2020), which becomes apparent in our study once again.

In view of the diversity in fluorochrome banding pattern, we attempted to resolve karyotype relationships by the UPGMA method. Identification of distinct subgroups has opened further scopes to complement marker assisted analysis of genetic diversity across varied range of Indian cultivars with valuable agronomic traits. Application of fluorochrome banding method has therefore helped to i) break the perception of an overall similar karyotype of cultivated Lentils as observed in Giemsa plates (Jha et al. 2015, 2017; Jha and Halder 2016) ii) serve as the chromosomal blueprint for cultivar discrimination, ii) statistically represent the status of chromosomal relationships, iii) highlight the uniqueness of certain Indian cultivars by means of unconventional banding pattern, and v) construct a fluorescent karyotype dataset of Indian Lentil cultivars.

\section{CONCLUSION}

Being a crop 'as old as agriculture' (Sandhu and Singh 2007), an exclusive chromosomal database of Lentils is essential to complement genomic research databases like Legume Information System (Dash et al. 2016) and KnowPulse (Sanderson et al. 2019). As an extension of our study involving Lentil cytogenetics, we have delved into the first molecular karyotypes of the country's native cultivars. Notably, the cultivars are hosted by world's second largest ex situ Lentil germplasm stock i.e. IIPR of NBPGR, the first being ICARDA (Muehlbauer and McPhee 2005; Coyne and McGee 2013). In future, molecular cytogenetic study of wild Lens species of India can be expected to strengthen the base of chromosomal evolution in Papilionoideae. In face of stern climatic changes that affect future cultivation, the Indian cultivars with interesting karyotype features and relationships can be fluently tested for performance and productivity. Thus, our findings complement traditional or marker assisted breeding and would undoubtedly bridge up the lacuna for a systematic chromosomal database of Indian Lentils.

\section{ACKNOWLEDGEMENTS}

TBJ acknowledges Dr. S. Dutta, Principal, Maulana Azad College, Kolkata for the facilities provided and Indian Institute of Pulses Research, Kanpur for providing certified Lentil cultivars. The grant was provided by the UGC, Govt. of India.

\section{REFERENCES}

Azani N, Babineau M, Bailey CD, Banks H, Barbosa AR, Pinto RB, et al. 2017. A new subfamily classification of the Leguminosae based on a taxonomically comprehensive phylogeny: The Legume Phylogeny Working Group (LPWG). Taxon. 66: 44-77.

Balyan HS, Houben A, Ahne R. 2002. Karyotype analysis and physical mapping of 18S-5.8 S-25S and 5S ribosomal RNA loci in species of genus Lens Miller (Fabaceae). Caryologia. 55(2): 121-128.

Barros e Silva AE, Guerra M. 2010. The meaning of DAPI bands observed after C-banding and FISH procedures. Biotech Histochem. 85(2): 115-125.

Bhattacharjee SK. 1953. Cytogenetics of Lens esculenta Moench. Caryologia. 5:159-166.

Bhowmick BK, Jha S. 2015. Differential heterochromatin distribution, flow cytometric genome size and meiotic behavior of chromosomes in three Cucurbitaceae species. Sci Hort. 193: 322-329.

Bhowmick BK, Jha S. 2019. Differences in karyotype and fluorochrome banding patterns among variations of Trichosanthes cucumerina with different fruit size. Cytologia. 84 (3):1-10.

Bonifácio EM, Fonsêca A, Almeida C, Dos Santos KG, Pedrosa-Harand A. 2012. Comparative cytogenetic mapping between the lima bean (Phaseolus lunatus L.) and the common bean (P. vulgaris L.). Theor Appl Genet. 124: 1513-1520.

Bortoleti KCDA, Benko-Iseppon AM, de Melo NF, Brasileiro-Vidal AC. 2012. Chromatin differentiation between Vigna radiata (L.) R. Wilczek and V. unguiculata (L.) Walp.(Fabaceae). Plant Syst Evol. 298: 689693.

Chaowen S, Liu J, Xiong Z, Song Y. 2004. Karyotype analysis of Psophocarpus tetragonolobus (L.) DC by chromosome banding and fluorescence in situ hybridization. Caryologia. 57: 387-394.

Chung MC, Lee YI, Cheng YY, Chou YJ, Lu CF. 2008. Chromosomal polymorphism of ribosomal genes in the genus Oryza. Theor Appl Genet. 116: 745-753.

Coyne C, McGee R. 2013. Lentil. In: Singh M, Upadhyaya HD, Bisht IS, editors. Genetic and genomic resources 
of grain legume improvement. Newnes: Elsevier Inc, p.157-180

Dash S, Campbell JD, Cannon EK, Cleary AM, Huang W, Kalberer SR, Karingula V, Rice AG, Singh J, Umale $\mathrm{PE}$, et al. 2016. Legume information system (LegumeInfo. org): a key component of a set of federated data resources for the legume family. Nucleic Acids Res. 44(D1): D1181-D1188.

Divashuk MG, Alexandrov OS, Razumova OV, Kirov IV, Karlov GI. 2014. Molecular cytogenetic characterization of the dioecious Cannabis sativa with an XY chromosome sex determination system. PloS one. 9: e85118.

Fernandes T, Rego LNAA, Nardy M, Yuyama PM, Vanzela ALL. 2009. Karyotype differentiation of four Cestrum species (Solanaceae) revealed by fluorescent chromosome banding and FISH. Genet Mol Biol. 32: 320-327.

Fuchs J, Strehl S, Brandes A, Schweizer D, Schubert I. 1998. Molecular-cytogenetic characterization of the Vicia faba genome - heterochromatin differentiation, replication patterns and sequence localization. Chromosome Res. 6: 219-230.

Fukui K. 1996. Plant chromosomes at mitosis. In: Fukui K, Nakayama S, editors. Plant chromosomes. Laboratory methods. Boca Raton, Tokyo: CRC Press, p. 1-18.

Galasso I. 2003. Distribution of highly repeated DNA sequences in species of the genus Lens Miller. Genome. 46:1118-1124.

Galasso I, Schmidt T, Pignone D. 2001. Identification of Lens culinaris ssp. culinaris chromosomes by physical mapping of repetitive DNA sequences. Chromosome Res. 9(3): 199-209.

Galasso MF, Maggiani M, Cremonini R, Pignone D. 1996. Chromatin characterization by banding techniques, in situ hybridization, and nuclear content in Cicer L. (Fabaceae). Genome. 39: 258-265.

Guerra M, Santos KGBD, E Silva AEB, Ehrendorfer F. 2000. Heterochromatin banding patterns in Rutaceae-Aurantioideae-a case of parallel chromosomal evolution. Am J Bot. 87(5): 735-747.

Jha T B, Saha P S, Jha S. 2020 A comparative karyo-morphometric analysis of Indian landraces of Sesamum indicum using EMA-giemsa and fluorochrome banding. Caryologia.73(2): 81-88.

Jha TB. 2019. Karyotype analysis from aerial roots of Piper nigrum based on Giemsa and fluorochrome banding. Cytologia. 84(4): 313-317.

Jha TB, Halder M. 2016. Searching chromosomal landmarks in Indian Lentils through EMA-based Giemsa staining method. Protoplasma. 253:1223-1231.
Jha TB, Mahanti A, Ghorai A. 2015. Karyotype analysis of Indian lentils through EMA-based Giemsa staining. Caryologia. 68:280-288.

Jha TB, Saha PS, Adak M, Jha S, Roy P. 2017. Chromosome morphometric analysis of Indian cultivars of Lens culinaris Medik. using EMA based Giemsa staining method. Caryologia. 70 (3) 270-283.

Jha TB, Yamamoto M. 2012. Application of EMA, fluorescence staining and FISH of rDNA in analysis of Aloe vera (L.) Burm. f. chromosomes. Bull Fac Agric Kagoshima Univ. 62:83-89.

Ladizinsky G. 1979. The origin of lentil and its wild gene pool. Euphytica. 28:179-187.

Ladizinsky G. 1999. Identification of lentil's wild genetic stock. Genet Resour Crop Ev. 46: 115-118.

Ladizinsky G, Weeden NF, Muehlbauer FJ. 1990. Tertiary trisomics in lentil. Euphytica. 51: 179-184.

Lavania UC, Lavania S. 1983. Karyotype studies in Indian pulses. Genet Agrar. 37:299-308.

Maragheh FP, Janus D, Senderowicz M, Haliloglu K, Kolano B. 2019. Karyotype analysis of eight cultivated Allium species. J Appl Genet. 60: 1-11.

Mehra RC, Butler MG, Beckman T. 1986. N banding and karyotype analysis of Lens culinaris. J Hered. 77:473474.

Mishra SK, Sharma B, Sharma SK. 2007. Genetics and cytogenetics of Lentil. In: Yadav SS, McNeil DL, Stevenson PC, editors. Lentil: An Ancient Crop for Modern Times. The Netherlands: Springer, p. 187-208.

Mondin M, Aguiar-Perecin ML. 2011. Heterochromatin patterns and ribosomal DNA loci distribution in diploid and polyploid Crotalaria species (Leguminosae, Papilionoideae), and inferences on karyotype evolution. Genome. 54: 718-726.

Moscone EA, Lein F, Lambrou M, Fuchs J, Schweizer D. 1999. Quantitative karyotyping and dual color FISH mapping of $5 \mathrm{~S}$ and $18 \mathrm{~S}-25 \mathrm{~S}$ rDNA probes in the cultivated Phaseolus species (Leguminosae). Genome. 42: 1224-1233.

Muehlbauer FJ, McPhee KE. 2005. Lentil (Lens culinaris Medik.). In: Singh RJ, Jauhar P, editors. Genetic resources and chromosome engineering and crop improvement. Vol 1, Grain legumes. Boca Raton London, New York: CRC, Taylor \& Francis Group; p. 219-230.

Naithani SP, Sarbhoy RK. 1973. Cytological studies in Lens esculenta Moench. Cytologia. 38:195-203.

Nandanwar RS, Narkhede MM. 1991. Intraspecific variation in karyotype of lentil. J Maharastra Agri Univ. 16:24-27.

Olanj N, Garnatje T, Sonboli A, Vallès J, Garcia S. 2015. The striking and unexpected cytogenetic diversity of 
genus Tanacetum L.(Asteraceae): a cytometric and fluorescent in situ hybridisation study of Iranian taxa. BMC Plant Biol. 15: 1-16.

Pedrosa-Harand A, de Almeida CC, Mosiolek M, Blair MW, Schweizer D, Guerra M. 2006. Extensive ribosomal DNA amplification during Andean common bean (Phaseolus vulgaris L.) evolution. Theor Appl Genet. 112: 924-933.

Raskina O, Barber JC, Nevo E, Belyayev A. 2008. Repetitive DNA and chromosomal rearrangements: speciation-related events in plant genomes. Cytogenet Genome Res. 120: 351-357.

Sanderson LA, Caron CT, Tan R, Shen Y, Liu R, Bett KE. 2019. KnowPulse: a web-resource focused on diversity data for pulse crop improvement. Front Plant Sci. 10 .

Sandhu JS, Singh S. 2007. History and Origin. In: Yadav SS, McNeil D, Stevenson PC. editors. Lentil: An ancient crop for modern times. The Netherlands: Springer, p 1-9.

Schweizer D. 1976. Reverse fluorescent chromosome banding with chromomycin and DAPI. Chromosoma. 58(4):307-324.

Sharma AK, Muhkopadhyay S. 1963. Karyotype constancy in different strains of Lens esculenta Moench as worked out through recent techniques. Indian Agric. 7:103-111.

She CW, Jiang XH. 2015. Karyotype analysis of Lablab purpureus (L.) sweet using fluorochrome banding and fluorescence in situ hybridisation with rDNA probes. Czech Journal of Genetics and Plant Breeding. 51: 110-116.

She CW, Jiang XH, Ou LJ, Liu J, Long KL, Zhang LH, et al. 2015. Molecular cytogenetic characterisation and phylogenetic analysis of the seven cultivated Vigna species (Fabaceae). Plant Biology. 17: 268-280.

She CW, Mao Y, Jiang XH, He CP. 2020. Comparative molecular cytogenetic characterization of five wild Vigna species (Fabaceae). Com. Cytogenet. 14: 243.

She CW, Wei L, Jiang XH. 2017. Molecular cytogenetic characterization and comparison of the two cultivated Canavalia species (Fabaceae). Com. Cytogenet. 11: 579.

Silvestri MC, Ortiz AM, Robledo GA, Lavia GI. 2020. Chromosome diversity in species of the genus Arachis, revealed by FISH and CMA/DAPI banding, and inferences about their karyotype differentiation. An Acad Bras Ciênc. 92.

Sinha SSN, Acharia SS. 1972. Karyotype analysis in some varieties of Lens culinaris. Cytologia. 37:673-683.

Souza MGC, Benko-Iseppon AM. 2004. Cytogenetics and chromosome banding patterns in Caesalpinioideae and Papilionioideae species of Pará, Amazonas, Brazil. Bot J Linn Soc. 144: 181-191.

Tadmor Y, Zamir D, Ladizinsky G. 1987. Genetic mapping of an ancient translocation in the genus Lens. Theor Appl Genet. 73:883-892.

Vanzela AL, Guerra M. 2000. Heterochromatin differentiation in holocentric chromosomes of Rhynchospora (Cyperaceae). Genet Mol Biol. 23(2): 453-456.

Weiss-Schneeweiss H, Schneeweiss GM. 2013. Karyotype diversity and evolutionary trend in angiosperms. In: Leitch IJ, Greilhuber J, Dolezel J, Wendel JF, editors. Plant genome diversity, vol. 2, physical structure, behavior and evolution of plant genomes. Vienna, Austria: Springer Verlag, p 209-230.

Yamamoto M. 2012. Recent progress on studies of chromosome observation in deciduous fruit trees. J Japan Soc Hort Sci. 81 (4): 305-313. 